\title{
A GAUSSIAN MIXTURE MODEL FOR CLASSIFYING THE HUMAN AGE USING DWT AND SAMMON MAP
}

\author{
${ }^{1}$ J. Nithyashri and ${ }^{2}$ G. Kulanthaivel \\ ${ }^{1}$ Department of Computer Science and Engineering, Sathyabama University, Chennai, India \\ ${ }^{2}$ Department of Electronics Engineering, NITTTR, Chennai, India
}

Received 2014-05-08; Revised 2014-05-23; Accepted 2014-11-28

\begin{abstract}
The appearance of a human face rigorously changes with respect to age that makes Age Classification as a more challenging task. The algorithms such as, K-Nearest Neighbor (K-NN), Support Vector Machine (SVM), Radial Basis Function (RBF), motivated many Face Researchers to focus their attention in classifying the human age into various age groups. The Classification rate produced by these existing algorithms is not significant indeed. In this study, Gaussian Mixture Models (GMM) is used for classifying the facial images into different age groups. A combination of Discrete Wavelet Transformation (DWT) and Sammon Map are used to extract the facial features. The performance of this approach is tested using Album-2 of MORPH database. A maximum classification rate of $99.52 \%$ is achieved in stage-1, whereas $99.46 \%$ is achieved in stage-2 using GMM. Also the accuracy achieved using Gaussian Mixture Model, is comparatively greater than K-NN.
\end{abstract}

Keywords: Pre-Processing, Gamma Correction, Contrast Enhancement, Facial Aging, Feature Extraction

\section{INTRODUCTION}

Age separated face recognition has wider applications, not only in the field of pattern recognition, but also in lot of other fields like Artificial Intelligence, Digital Image Processing, Human Computer Interaction, Machine Learning and so on. However, many researchers proposed a variety of approaches and algorithms in face recognition, but very few researchers turned their attention towards age separated faces. Age invariant face recognition, Age Estimation and Classification of Human Age are the challenging areas of research. The human age are classified into various age groups like Child, Adolescence, Adult, Middle-aged Adult and Senior Adult etc. The very first work in classifying the human age is introduced by (Kwon and Lobo 1999), where they classified the age groups into Babies, Young,
Adult and Senior Adult. In this study, we introduce a novel approach in classifying the human age into various age groups. We also used an Image Enhancement technique to normalize the facial images, which was proposed by (Tan and Triggs, 2010). It includes Gamma Correction, DoG Filtering and Contrast Enhancement to enhance the facial images. Then, the normalized image is transformed, using Discrete Wavelet Transformation which is used to extract the required facial features. The dimensions of the image are not completely reduced. Consequently, a Sammon Map is used to diminish the dimensions of the image further. We further used a Gaussian Mixture Model (GMM) to classify the images into various age groups. In this study, the images are randomly selected from Album-2 of MORPH database, presented in (Ricanek and Tesafaye, 2006).

The supporting contents of this manuscript are planned as follows: Chapter 2, confer about related Corresponding Author: J. Nithyashri, Department of Computer Science and Engineering, Sathyabama University, Chennai, India 
works, Chapter 3, focuses the overview of the proposed work, Chapter 4, shows the results and discussions and Chapter 5 gives the conclusion and future directions.

\section{PREVIOUS WORK}

The age groups were determined using, various algorithms like Radial Basis Function (RBF), presented by (Yazdi et al., 2012), Back Propagation Network presented by (Mehdi et al., 2009) and Support Vector Machine (SVM)-Sequential Minimum Optimization (SMO), which includes mathematical techniques related to facial features. A combination of Discrete Wavelet Transform and Gradient Orientation pyramid for extracting the facial features was proposed by (Saeid and Leila, 2012). Subsequently, the dimensions of the images are further reduced using Principal Component Analysis (PCA). Furthermore, the recognition phase was done by computing the distance using Euclidean Distance. Now-a-days, Discrete Wavelet Transforms and Neural Network is used to identify the epilepsy disorder. Also the classification of Electroencephalograph (EEG) is done using Back Propagation Network presented by (Kumar and Kasthuri, 2014).

Wen-Bing et al. (2001) developed a classification technique based on facial features, in which they classified the age groups into four categories. A combination of two distance measures such as, Cosine and Euclidean distance methods were used to normalize the distance, for providing greater accuracy than using one method, which was presented by (Zou, 2008). Zhan and Ogunbona (2011) developed a Non-negative Matrix Factorization (NMF), for estimating the human age. The age-group of a face image is estimated, based on the learned age subspaces. However, the age progression algorithms was developed, based on Age Estimation methods, as investigated in (Geng et al., 2007). To improve the accuracy of Age Estimation, a combination of Active Appearance Model (AAM) and Support Vector Machine was introduced by (Luu et al., 2009). The most prominent algorithm namely, the Principal Component Analysis (PCA) was used in reducing the dimensions of the images. After extracting the facial features, the Support Vector Machine (SVM) had been trained, to classify the facial images into different age groups which was proposed by (Liu et al., 2012). A Gaussian Mixture Model (GMM)-based human face recognition technique robust to illumination variations was proposed by (Mitra, 2012) and the misclassification error rate is also reduced. An innovative approach was introduced by (Kumar et al., 2013) in which topological features were used to classify the age groups into five categories.

\section{PROPOSED WORK}

The proposed work for age classification is illustrated in the Fig. 1. The input facial images are improved using different stages of Pre-processing techniques. As a result, the image is said to be normalized and is given as an input to next phase, in which the facial features are extracted using DWT. The dimensions of the image are further reduced using Sammon Map and hence it simplifies the classification phase. The age groups are classified using Gaussian Mixture Model.

\subsection{Pre-Processing}

A sequence of pre-processing technique is introduced, to enhance the facial images. Initially, the gray-scaled images are enhanced using Gamma Correction, where the brightness of the images is adjusted. Secondly, the shading effects are completely removed using DoG filtering. Finally, the contrast of the images is adjusted by eliminating the intensity variations in the images. The pre-processed or normalized images are given as input to the next phase namely the feature extraction.

\subsection{Feature Extraction Using DWT and Sammon Map}

The Discrete Wavelet Transformation (DWT) is a conservative means of feature extraction in Image Processing and Computer Vision. There are several applications of Wavelet Transforms such as Image Compression, Face Recognition and Age Prediction. Wavelet Transforms are primarily used in various applications because of reduced complexity. Discrete Wavelet Transform is achieved by dilating/translating the mother wavelet $\psi(x)$ and it is shown in the following Equation 1:

$$
\Psi_{p, q}(x)=\frac{1}{\sqrt{p}} \Psi\left(\frac{x-q}{p}\right)
$$

Here, $\sqrt{p}$ is the normalization factor, whereas, $p$ and $q$ are the scaling parameter and translation parameter respectively. The Discrete Wavelet Transformation is achieved by filtering the signal through a series of digital filters at various scales. 


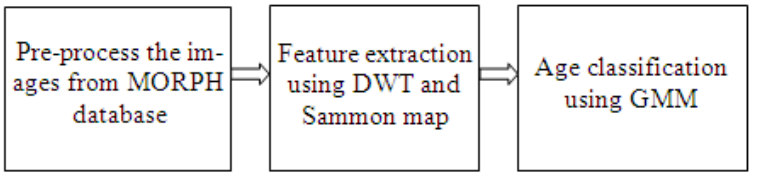

Fig.1. Block Diagram of the proposed work

The dimensions of the image are further reduced, using a non-linear technique known as Sammon Map. Sammon's non-linear mapping, was presented by (Yang, 2004) and is an iterative process of projecting a higher dimensional space into lower dimensional space. As a result the image would be geometrically congruent to the original image. This is called an isometric projection. The comparison between two geometric objects means, that the corresponding inter-point distances are equal. Instead of trying to achieve equality between corresponding inter-point distances, we can minimize the difference between corresponding inter-point distances. This is one goal of the Sammon mapping algorithm.

\subsection{Classification of Age Groups Using Gaussian Mixture Model (GMM)}

Gaussian Mixture Model is a parametric framework suitable for modelling the human faces and is proposed in (Mitra, 2012). It is a mixture of numerous Gaussian distributions and consequently represents different subclasses in one class. The general form of Probability Density Function is Equation 2:

$$
g(x)=\sum_{i=1}^{n} w_{i} \cdot N\left(x ; \mu_{i} \Sigma_{i}\right)
$$

Here $n$ represents the number of components and $w_{i}$ represents the weights of Gaussian Component Equation 3:

$$
\sum_{i=1}^{n} w_{i}=1, \text { and } \forall_{i}: w_{i}>=0
$$

$N\left(x ; \mu_{i}, \Sigma_{i}\right)$ represents the Probability Density Function of Normal Distribution and $\mu_{i}, \Sigma_{i}$ are the parameters of the Gaussian distribution representing Mean and Covariance Matrix respectively. The Gaussian Mixture Density is collection of all parameters like mean, mixture weights and the covariance matrix. Each and every Gaussian Model has its own Covariance Matrix, Mean, which is to be estimated separately. However, selecting the Gaussian Mixture Components plays a vital role in classification domain in which, each component represents a separate class. There are various algorithms stating, how to choose the number of components and is presented in (Figueiredo and Jain, 2002). When there are many components then there exists difficulty in fitting the data, whereas a mixture with small number of components may not be flexible to estimate the true fundamental model.

\section{EXPERIMENTAL WORK}

An experimental work is done using 1250 images, arbitrarily selected from Album 2 of MORPH database which is proposed in (Ricanek and Tesafaye, 2006). It includes 500 images of Adolescence and Adult, 250 images of Senior Adult. Initially, the face images are cropped to a size of about $128 \times 128$. The face images is normalized using 3 -stage pre-processing techniques. Further, the facial features are extracted using Discrete Wavelet Transformation, namely Haar, Symlet, Deubechies and Bi-orthogonal wavelets at level-1, level2 and level-3. The implementation of this study is done using MATLAB. The Fig. 2 shows the longitudinal image database namely, the MORPH database cropped to a size of $128 \times 128$.

The cropped faces are converted into gray scale images. A three-stage of normalization technique is used to enhance the facial images. At first, the brightness of the image is improved using Gamma Correction, in which the brightness of the image is adjusted. Secondly, the edges of the images are exactly obtained by applying DoG filter and finally, the contrast enhancement is done so as to adjust the contrast of the image. Actually, threestages of pre-processing are done, in order to get a normalized image. The facial features are extracted by transforming the normalized images, using Discrete Wavelet Transforms like Haar, Symlet, Deubechies and Bi-orthogonal at level-1, level-2 and level-3. However, the dimensions of the images are not completely reduced; further, we applied a dimension reduction technique, called the Sammon Map. A two-stage classification technique is used, in which stage-1 classifies whether the given facial image is an adolescent or not. On the other hand, stage- 2 classifies whether the facial image is an adult or senior adult. We use $g=4$, $8,16,32,64$ for all the wavelets and at all levels. The Table 1 shows the classification rate of stage-1 using Discrete Wavelets at level-3. The training and testing samples are varied for 10 different times and the results are tabulated. In addition to that, Table 2 shows the classification rate of stage-2. The maximum classification rate of stage 1 and stage 2 is about 99.52 and $99.46 \%$ respectively. The performance of stage1classification is depicted in Fig. 3, whereas the performance of stage-2 classification is depicted in the Fig. 4. We also compared the obtained classification rate with K-NN and the Fig. 5 shows the comparative analysis of these approaches. 
J. Nithyashri and G. Kulanthaivel / Journal of Computer Science 10 (11): 2292.2298, 2014

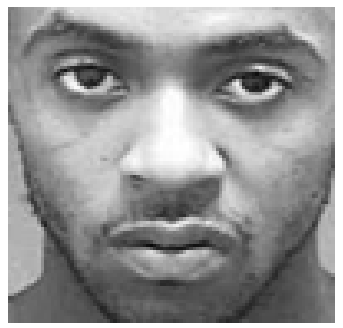

(a)

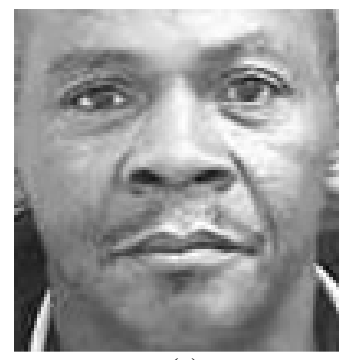

(a)

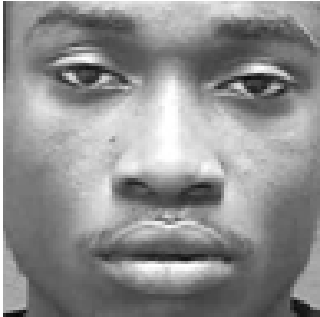

(b)

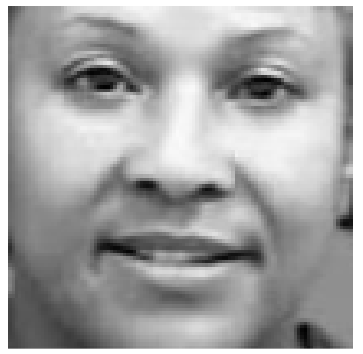

(b)

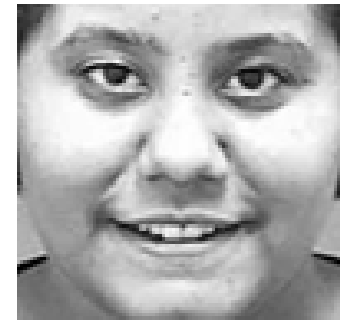

(c)

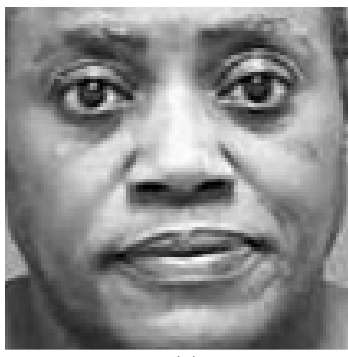

(c)

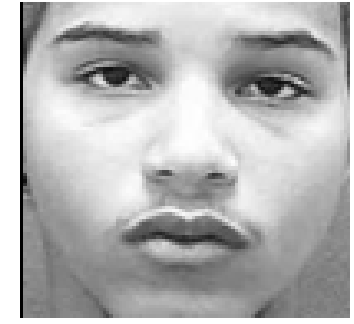

(d)

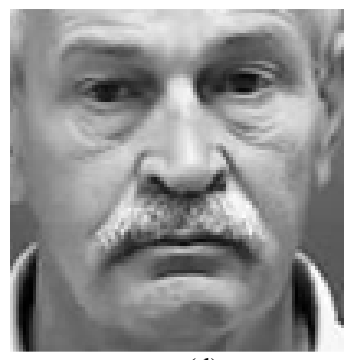

$\mathrm{s}(\mathrm{d})$

Fig. 2. Cropped images of size $128 * 128$ from MORPH database

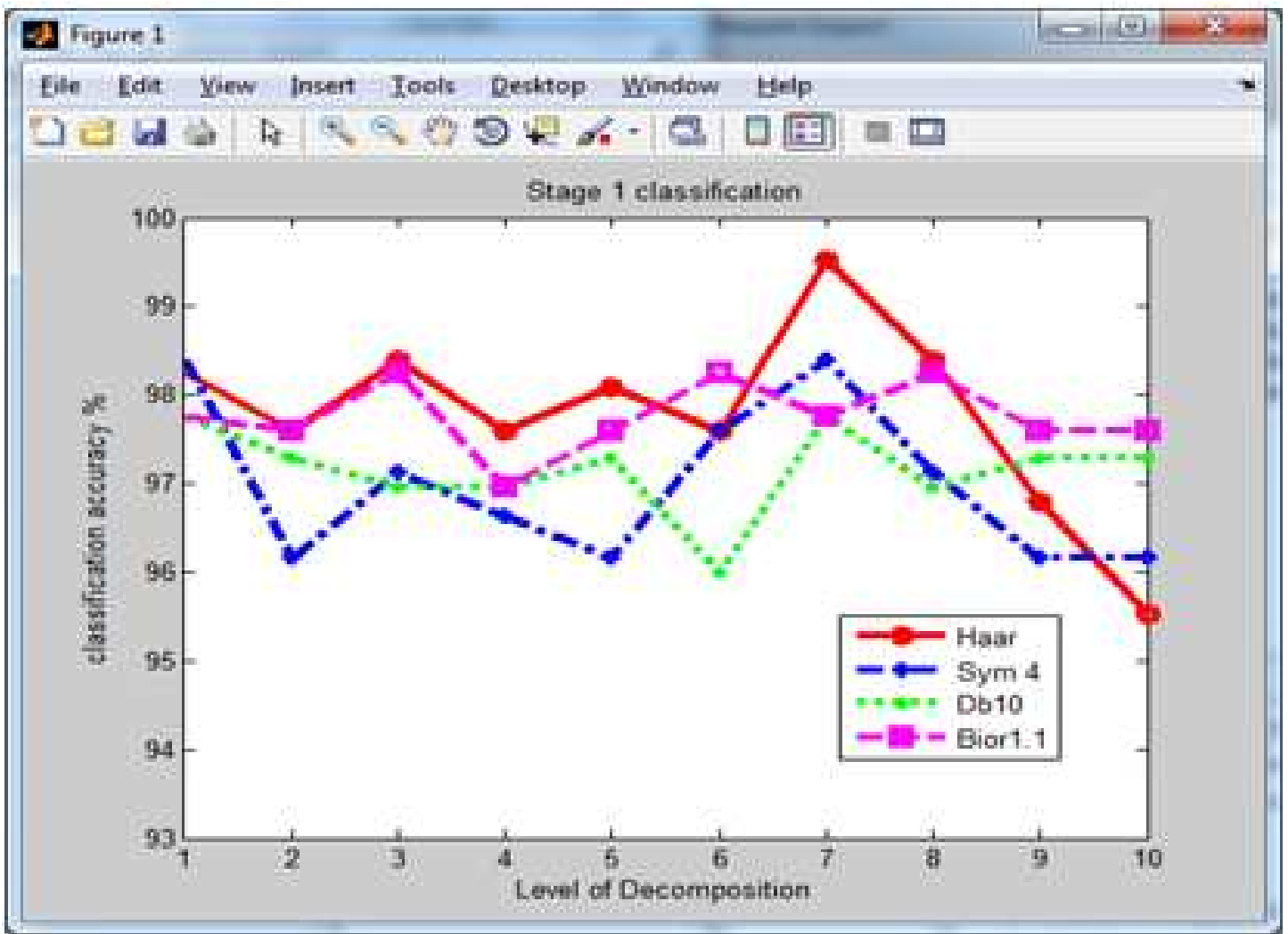

Fig. 3. Performance of stage-1 classification 


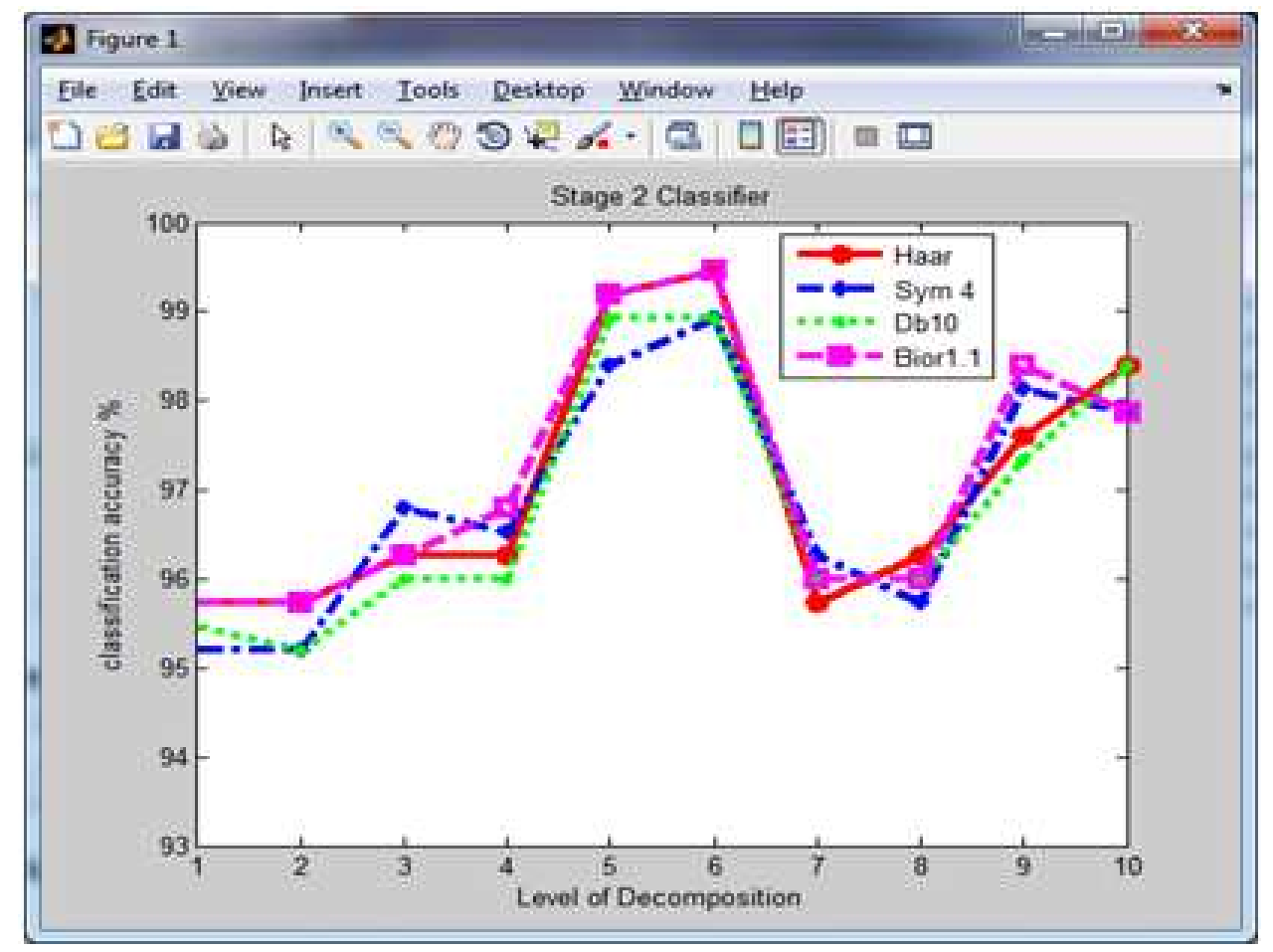

Fig. 4. Performance of stage-2 classification

Performance of GMM

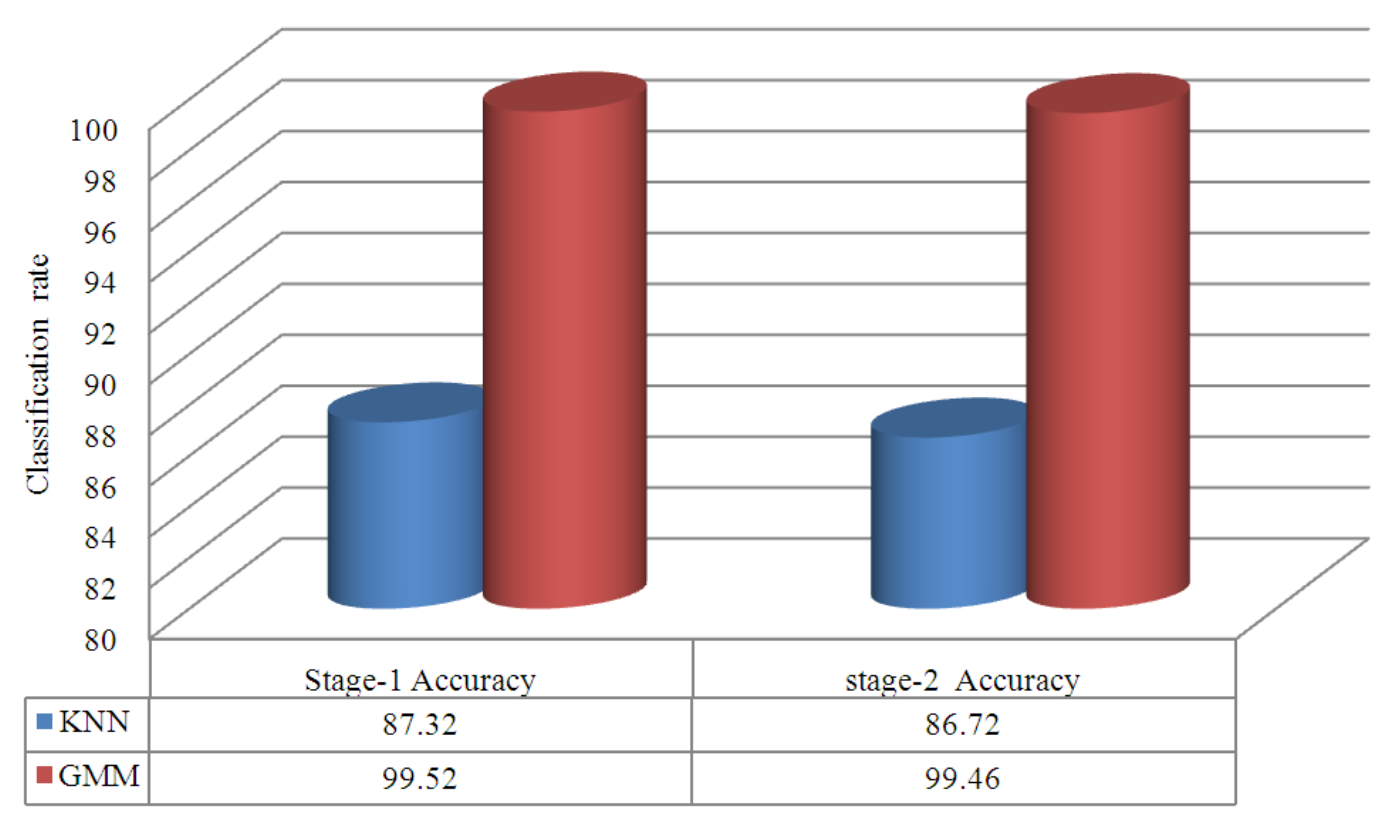

Fig. 5. Comparative analysis of different approaches 
Table 1. Stage 1 classification using DWT+SAMMON MAP+GMM Accuracy in \%

\begin{tabular}{lllll} 
Iteration & Haar & Sym $(4)$ & db $(10)$ & bior 1.1 \\
\hline 1 & 98.24 & 98.40 & 97.76 & 97.76 \\
2 & 97.60 & 96.16 & 97.28 & 97.60 \\
3 & 98.40 & 97.12 & 96.96 & 98.24 \\
4 & 97.60 & 96.64 & 96.96 & 96.96 \\
5 & 98.08 & 96.16 & 97.28 & 97.60 \\
6 & 97.60 & 97.60 & 96.00 & 98.24 \\
7 & 99.52 & 98.40 & 97.76 & 97.76 \\
8 & 98.40 & 97.12 & 96.96 & 97.24 \\
9 & 96.80 & 96.16 & 97.28 & 97.60 \\
10 & 95.52 & 96.16 & 97.28 & \\
\hline
\end{tabular}

Table 2. Stage 2 classification using DWT+SAMMON MAP+GMM

\begin{tabular}{|c|c|c|c|c|}
\hline \multicolumn{5}{|c|}{ Accuracy in $\%$} \\
\hline Iteration & Haar & Sym (4) & $\mathrm{db}(10)$ & bior1 \\
\hline 1 & 95.73 & 95.20 & 95.46 & 95.73 \\
\hline 2 & 95.73 & 95.20 & 95.20 & 95.73 \\
\hline 3 & 96.26 & 96.80 & 96.00 & 96.26 \\
\hline 4 & 96.26 & 96.53 & 96.00 & 96.80 \\
\hline 5 & 99.20 & 98.40 & 98.93 & 99.20 \\
\hline 6 & 99.46 & 98.93 & 98.93 & 99.46 \\
\hline 7 & 95.73 & 96.26 & 96.00 & 96.00 \\
\hline 8 & 96.26 & 95.73 & 96.00 & 96.00 \\
\hline 9 & 97.60 & 98.13 & 97.33 & 98.40 \\
\hline 10 & 98.40 & 97.86 & 98.40 & 97.86 \\
\hline
\end{tabular}

\section{CONCLUSION}

In this study, we developed a different approach, in which the face images are enhanced by a sequence of pre-processing techniques. To extract the facial features, the normalized images are transformed using DWT. The dimensions of the image are further reduced using Sammon Map. Then GMM is used for classifying the facial images into different age-groups and tested using Album 2 of MORPH Database. An utmost classification rate of $99.56 \%$ is achieved in stage- 1 and $99.46 \%$ is achieved in stage- 2 classification. It is concluded that the performance of DWT + Sammon Map + GMM performs better than that of the DWT + Sammon Map + KNN. The problems encountered in our work are of two kinds and they are (i) the child images are not available in the database and hence the classification cannot be done for images below 12 years and (ii) the classification rate is not significant for noisy images. The proposed work can be extended by training different images of Google Database, FERET database, Iranian Face Database and other reputed databases. The work can also be tested using other kind of Discrete Wavelet transformations at various levels.

\section{ACKNOWLEDGEMENT}

The researchers would like to thank Dr. Karl Ricanek Jr, Department of Computer Science, University of North Carolina, Wilmington, for the support of MORPH database.

\section{REFERENCES}

Saeid, F. and A.H. Leila, 2012. Wavelet based age invariant face recognition using gradient orientation. Proceedings of the International Conference on Advances in Computer and Electrical Engineering, Nov. 17-18, pp: 13-16. 
Figueiredo, M. and A.K. Jain, 2002. Unsupervised learning of finite mixture models. IEEE Trans. Pattern Anal. Mach. Intelli., 24: 381-396. DOI: 10.1109/34.990138

Geng, X., Z.H. Zhou and K. Smith-Miles, 2007. Automatic age estimation based on facial aging patterns. IEEE Trans. Pattern Anal. Mach. Intelli., 29: 2234-2240. DOI: 10.1109/TPAMI.2007.70733

Kumar, S.T.S. and N. Kasthuri, 2014. Determination of epileptic disorder with discrete wavelet transforms and neural network classifier. J. Comput. Sci., 10: 66-72. DOI: 10.3844/jcssp.2014.66.72

Kumar, V., J.S. Kiran and H. Chandana, 2013. An effective age classification using topological features based on compressed and reduced grey level model of the facial skin. Int. J. Image Graph. Sig. Process., 6: 9-17. DOI: $10.5815 /$ /ijigsp2014.01.02

Kwon, Y.H. and N.D. V. Lobo, 1999. Age classification of facial images. J. Comput. Vision Image Understand., 74: 1-21. DOI: 10.1006/CVIU.1997.0549

Liu, L., J. Liu and J. Cheng, 2012. Age-Group Classification of Facial Images. Proceedings of the 11th International Conference on Machine Learning and Applications, Dec. 12-15, IEEE Xplore Press, Boca Raton, FL, pp: 693-696. DOI: 10.1109/ICMLA.2012.129

Mehdi, L., A. Solimani, A. Dragazany, H. Afzal and M. Bandarabadi, 2009. Combining wavelet transforms and neural networks for image classification. Proceedings of the IEEE 41st South Eastern Symposium on System Theory, Mar. 1517, IEEE Xplore Press, Tullahoma, TN, pp: 4448. DOI: $10.1109 /$ SSST.2009.4806819

Luu, K., J.R. Ricanek, K. Suen, C.Y. and T.D. Bui, 2009. Age estimation using active appearance models and support vector machine regression. Proceedings of the IEEE International Conference on Biometrics: Theory Applications and System, Sept. 28-30, pp: 314-318. DOI: 10.1109/BTAS.2009.5339053
Mitra, S., 2012. Gaussian mixture model for human face recognition under illumination variations. $J$. Applied Math., 3: 2071-2079. DOI: 10.4236/am.2012.312A286

Ricanek, K. and T. Tesafaye, 2006. MORPH: A longitudinal image database of normal adult ageprogression. Proceedings of the IEEE 7th International Conference on Automatic face and Gesture Recognition, Apr. 10-12, IEEE Xplore Press, Southampton, pp: 341- 345. DOI: 10.1109/FGR.2006.78

Tan, X. and B. Triggs, 2010. Enhanced local texture feature sets for face recognition under difficult lighting conditions. IEEE Trans. Image Process., 19: 1635-1649. DOI: 10.1109/TIP.2010.2042645

Wen-Bing, H., L. Cheng-Ping and C. Chun-Wen, 2001. Classification of age groups based on facial features. J. Sci. Eng., 4: 183-192.

Yang, L., 2004. Nonlinear mapping using geodesic distances. Proceedings of IEEE 17th International Conference on Pattern Recognition, Aug. 23-26, IEEE Xplore Press, pp: 303-306. DOI: 10.1109/ICPR.2004.1334180

Yazdi. M., S. Mardani-Samani, M. Bordbar and R. Mobaraki, 2012. Age classification based on RBF Neural network. Canadian J. Image Process. Comput. Vision, 3: 38-42. DOI: 10.5120/15780-4480

Zhan, C. and W.P. Ogunbona, 2011. Age estimation based on extended non-negative matrix factorization. Proceedings of the 13th IEEE International Workshop on Multimedia Signal Processing, Oct. 17-19, IEEE Xplore Press, Hangzhou, pp: 1-6. DOI: 10.1109/MMSP.2011.6093779

Zou, B.J., 2008. Shape-based trademark retrieval using cosine distance method. Proceedings of the 8th International Conference on Intelligent Systems Design and Applications, Nov. 26-28, IEEE Xplore Press, Kaohsiung, pp: 498-504. DOI: $10.1109 /$ ISDA.2008.161 